\title{
Effect of Electrophoretically Deposited Graphene Nanoplatelets on Flexural Properties of Carbon Fabric/Epoxy Laminated Composites
}

\author{
Ervina Junaidi, ${ }^{1,2}$ Mariatti Jaafar, ${ }^{1 *}$ Sinin Hamdan $^{2}$ and Hitoshi Takagi ${ }^{3}$ \\ ${ }^{1}$ School of Materials and Mineral Resources Engineering, Universiti Sains Malaysia, \\ Engineering Campus, 14300 Nibong Tebal, Pulau Pinang, Malaysia \\ ${ }^{2}$ Department of Mechanical and Manufacturing Engineering, Faculty of Engineering, \\ Universiti Malaysia Sarawak, 94300 Kota Samarahan, Sarawak, Malaysia \\ ${ }^{3}$ Department of Mechanical Science, Graduate School of Technology, \\ Industrial and Social Sciences, Tokushima University, Japan \\ *Corresponding author: mariatti@usm.my
}

Published online: 25 December 2019

To cite this article: Junaidi, E. et al. (2019). Effect of electrophoretically deposited graphene nanoplatelets on flexural properties of graphene nanoplatelets/carbon fabric/epoxy hybrid laminated composites. J. Phys. Sci., 30(Supp. 2), 91-102, https://doi.org/10.21315/ jps2019.30.s2.8

To link to this article: https://doi.org/10.21315/jps2019.30.s2.8

\begin{abstract}
In this study, the graphene nanoplatelets (GNP)/carbon fabric (CF)/epoxy (EP) hybrid laminated composites were fabricated via electrophoretic deposition (EPD) followed by hand lay-up assisted vacuum bagging technique. GNP were dispersed in two different colloidal suspensions, i.e., distilled water $(D W)$ and $N$-dimethyformamide $(D M F)$, and the effect of EPD GNP onto CF surface were investigated. The finding indicated that the electrophoretic mobility (EM) of GNP is slightly slower in DMF due to the higher viscosity of DMF (0.92 mPa.s) compared to DW $(0.89 \mathrm{mPa} . \mathrm{s})$. Zeta $(\xi)$ potential of GNP were slightly higher in DMF, revealing the non-polar behaviour of GNP being easily dispersed in DMF due to its low polar and hydrogen-bonding strength. However, the higher current intensity was observed for the GNP-DW suspension, implying higher dielectric constant of DW adequate for effective deposition of GNP onto CF surface as confirmed via field-emission scanning electron microscopy (FESEM). The GNP-DMF/ $C F / E P$ composites exhibit the highest flexural properties compared to GNP-DW/CF/EP composites. These findings proved the colloidal dispersibility of GNP in DW and DMF suspensions, respectively used in EPD govern the flexural properties of $G N P / C F / E P$ hybrid laminated composites.
\end{abstract}

Keywords: Graphene nanoplatelets, carbon fibre, electrophoretic deposition, colloidal stability, flexural properties 


\section{INTRODUCTION}

Advanced structural fibre such as carbon fibres (CF) with their exceptional mechanical properties have been extensively used as reinforcements in high performance structural composite applications. However, there are some limitations with advanced composites due to the compatibility between $\mathrm{CF}$ and matrix. A nonpolar, stable and smooth graphitic surface of CF result in weak adhesion and poor bonding formed between $\mathrm{CF}$ and matrix. Many attempts have been highlighted on enhancing material properties via developing graphene nanoplatelet (GNP) based hybrid composites due to the fact that hybrid fibre-GNP structures can considerably improve the composite performance. ${ }^{1,2}$

Several methods have been used to create hybrid fibre-GNP structures such as electrophoretic deposition (EPD), chemical vapour deposition (CVD), dipping and spraying. ${ }^{3,4}$ The EPD, dipping and spraying methods involve the liquid phase medium and are based on physical interactions between filler and the fibre surface. Among these methods, EPD has shown to be effective for deposition of GNP onto CF surface due to low cost, process simplicity, uniformity of deposits and lower applied voltage requirements. ${ }^{5}$

The aim of the present work is to investigate the effect of different suspensions for distilled water (DW) and N-dimethylformamide (DMF) on the dispersibility and stability of GNP in colloidal suspension. Quantitative estimation of GNP suspension stability in different suspension (DW and DMF) was performed by measuring electrophoretic mobility (EM) and the zeta $(\xi)$ potential of the GNP suspension with different $\mathrm{pH}$ values. The relationship between the current intensity and the morphology of deposited GNP-DW and GNP-DMF were further investigated and correlated with the flexural properties of GNP/CF/EP hybrid laminated composites.

\section{EXPERIMENTAL}

\subsection{Materials}

GNP with an average flake-like particle diameter and thickness of $15 \mu \mathrm{m}$ and 11-15 nm, respectively were supplied by Skyspring Nanomaterials, United States. Figures 1( $a$ and $b$ ) display the field emission scanning electron microscopy (FESEM) and transmission electron microscopy (TEM) images of the as-received GNP. The FE-SEM image reveals the morphology of the GNP showing smooth thin layers of graphene sheets stacked on top of each other. As shown from TEM image, there are few darker black shades in the structure of GNP, proving that 
GNP are wrinkled at certain areas due to GNP form aggregates. Figures 1(c and d) show the digital and FESEM images of CF and CF filament, respectively. The plain-weave CF (3000-multifilament) was supplied by Fiber Glast Developments Corporation, United States. Epoxy resin, D.E.R. ${ }^{\text {TM }} 332$ with a density of $1.16 \mathrm{~g} \mathrm{~cm}^{-3}$ and curing agent, polyetheramines D-230 with a density of $0.946 \mathrm{~g} \mathrm{~cm}^{-3}$ were supplied by Penchem Technologies Sdn. Bhd., Malaysia. $\mathrm{N}$-dimethyformamide (DMF), concentrated nitric acid $\left(\mathrm{HNO}_{3}\right)$ of $65 \%$ and potassium hydroxide $(\mathrm{KOH})$ were supplied by Merck Sdn. Bhd., Malaysia.
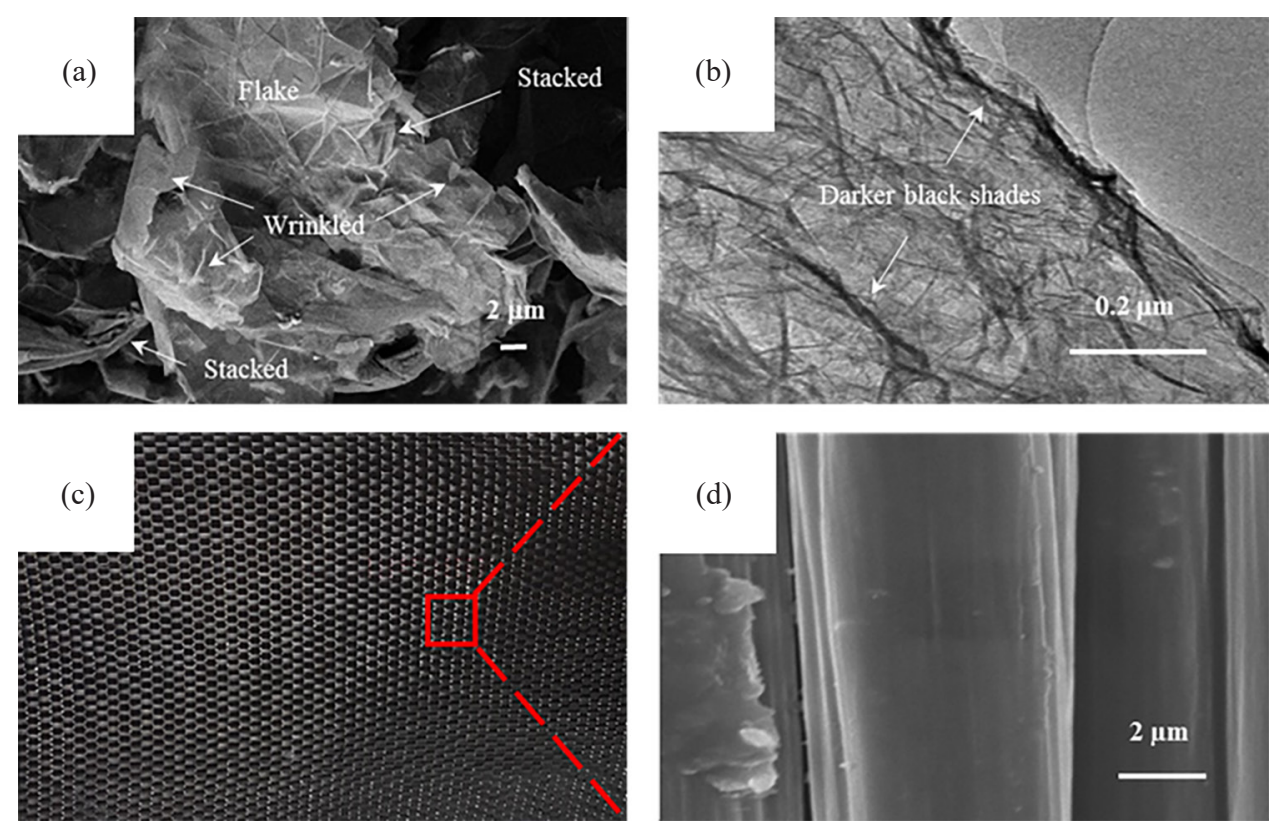

Figure 1: Illustrations of (a, c) FESEM, and (b, d) TEM images of GNP (with magnifications of $2.5 \mathrm{KX}$ for (a) and $1.05 \mathrm{MX}$ for (b). Illustration (c) shows digital image of CF, and (d) is FESEM image of CF filament with magnification of $5 \mathrm{KX}$.

\subsection{Fabrication of Hybrid Laminated Composites}

GNP suspensions (0.05 $\mathrm{mg} \mathrm{ml}$ ) were prepared by dispersing GNP in DW and DMF suspensions, respectively. The suspensions were ultrasonicated individually, using a Hielscher-UP200S Ultrasonicator for $20 \mathrm{~min}$ to form a stable suspension. The EPD of GNP in different suspensions (DW and DMF) were performed at their control $\mathrm{pH}$ (6.59 and 6.45) with the presence of fixed applied voltage $(20 \mathrm{~V})$ and deposition time ( $5 \mathrm{~min}$ ) based on optimised condition achieved from previous work. ${ }^{6}$ Figure 2 schematically illustrates the EPD of GNP onto the CF surface. 
Both the electrodes (stainless steel and CF) were fixed in parallel (at a distance of $1.5 \mathrm{~cm}$ ). The current was recorded as a function of deposition time. In the EPD process, the migration direction of the filler in suspension during EPD was controlled by the filler surface charge, where the positively charged filler was attracted to the cathode electrode and vice versa. ${ }^{5}$ The GNP-coated CF $(120 \mathrm{~mm}$ height $\times 120 \mathrm{~mm}$ length) dispersed separately in DW and DMF were both dried at room temperature $(24 \mathrm{~h})$ followed by drying in the oven at $100^{\circ} \mathrm{C}(24 \mathrm{~h})$ and $153^{\circ} \mathrm{C}$ (24 h), respectively.

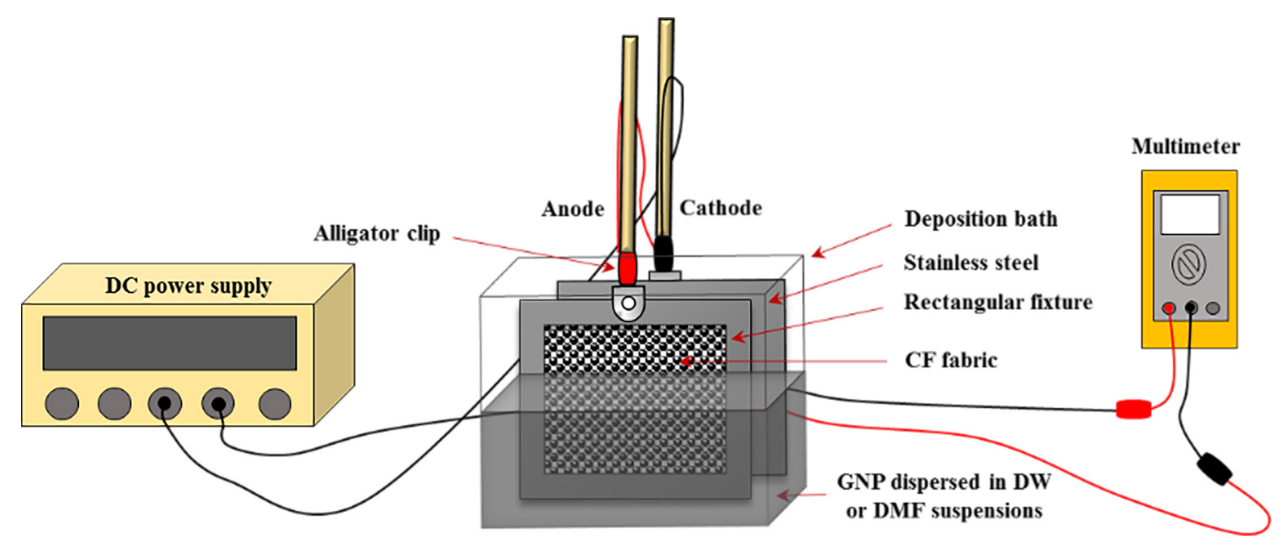

Figure 2: Schematic illustration of EPD GNP onto CF surface.

The fabrication of 3-ply GNP-coated CF (CF-GNP/EP) and the controlled sample (CF/EP) hybrid laminated composites were performed via hand lay-up assisted vacuum bagging technique. Three pieces of GNP-coated CF and controlled samples $(120 \mathrm{~mm}$ height $\times 120 \mathrm{~mm}$ length $)$ were used. Pre-calculated amounts of CF, EP and GNP were weighted with the ratio of CF to EP fixed at 60:40. The EP and the curing agent were mixed at 100:32 ratio prior to hand lay-up process. The hybrid laminated composites were then fabricated via hand lay-up method and then consolidated for about 45 min using vacuum bagging technique. The fabricated composite was left overnight at room temperature and then subject to a post-curing process where the composite was heated at a temperature of $80^{\circ} \mathrm{C}(2 \mathrm{~h})$ and then post-cured at $125^{\circ} \mathrm{C}(3 \mathrm{~h})$.

\subsection{Characterisations}

FESEM (Zeiss-SUPRA-35VP) and TEM (Philips-CM12) were used to examine the morphology of the GNP. Also, FESEM was used to observe the surface morphology of the CF filament and the GNP-coated CF. The Zetasizer-nano analysis $\xi$ potential and electrophoretic mobility (EM) of the GNP-DW and GNP- 
DMF were measured using the Zetasizer-Nano Instrument (Nano-ZS-ZEN3600, Malvern, UK). The $\mathrm{pH}$ of GNP suspension was adjusted between 2 and 12 by the addition of suitable quantities of aqueous $\mathrm{HNO}_{3}$ and $\mathrm{KOH}$ solutions, respectively. ${ }^{5}$ Flexural test of the hybrid laminated composites was conducted following ASTM D790-02 using universal testing machine (Instron 3366, United States).

\section{RESULTS AND DISCUSSION}

\subsection{Electrophoretic Mobility and Zeta Potential}

Figure 3 displays the results for the EM and $\xi$ potential as a function of $\mathrm{pH}$ for the GNP dispersion in the DW and DMF suspensions, respectively. It can be observed from Figure 3(a) that the EM of GNP-DW and GNP-DMF become more negative and travel rapidly as the $\mathrm{pH}$ increases and this observation may be associated with deprotonation of the functional groups. The EPD of GNP-DW and GNP-DMF suspensions at their control $\mathrm{pH}$ exhibits EM of $-2.75 \mu \mathrm{m} . \mathrm{cm} / \mathrm{V} . \mathrm{s}(\mathrm{pH}=6.59)$ and $-1.38 \mu \mathrm{m} . \mathrm{cm} / \mathrm{V} . \mathrm{s}(\mathrm{pH}=6.45)$, respectively. The findings demonstrated the EM of GNP-DMF was slightly slower compared to GNP-DW probably due to the higher viscosity of DMF (0.92 mPa.s) compared to DW (0.89 mPa.s). A previous study reported that an increased in suspension viscosity, limiting the EM of filler in suspension. ${ }^{7}$

The $\xi$ potential for the GNP-DW and GNP-DMF suspensions is shown in Figure 3(b). Theoretically, the $\xi$ potential of filler is dependent on the $\mathrm{pH}$ of the filler suspension as the $\mathrm{pH}$ affects the degree of ionisation as well as the stability of the suspension. ${ }^{8}$ Both the GNP-DW and GNP-DMF suspensions have strongly negative $\xi$ potential values as $\mathrm{pH}$ of the suspension increased and formed stable dispersions in the $\mathrm{pH}$ range of $6-12$ with the highest mean $\xi$ potential value of $-54.2 \mathrm{mV}$ and $-57.9 \mathrm{mV}$, respectively at $\mathrm{pH}=12$. The observation showed that the deprotonation of the functional group is intensely associated with the $\mathrm{pH}$ of the suspension, leading to the highly negative $\xi$ potential and subsequently improved the stability of GNP-DW and GNP-DMF suspensions. ${ }^{9}$ At the control $\mathrm{pH}$ of 6.59, the GNP-DW suspension shows a negative mean $\xi$ potential of $-35.0 \mathrm{mV}$. The mean $\xi$ potential of GNP at their control $\mathrm{pH}$ was slightly higher in DMF which was $-37.4 \mathrm{mV}$. The finding revealed that the non-polar behaviour of untreated GNP is easily dispersed in polar aprotic solvent (DMF) compared to polar protic solvent (DW) possibly due to their low polar and hydrogen-bonding strength. ${ }^{10}$ 


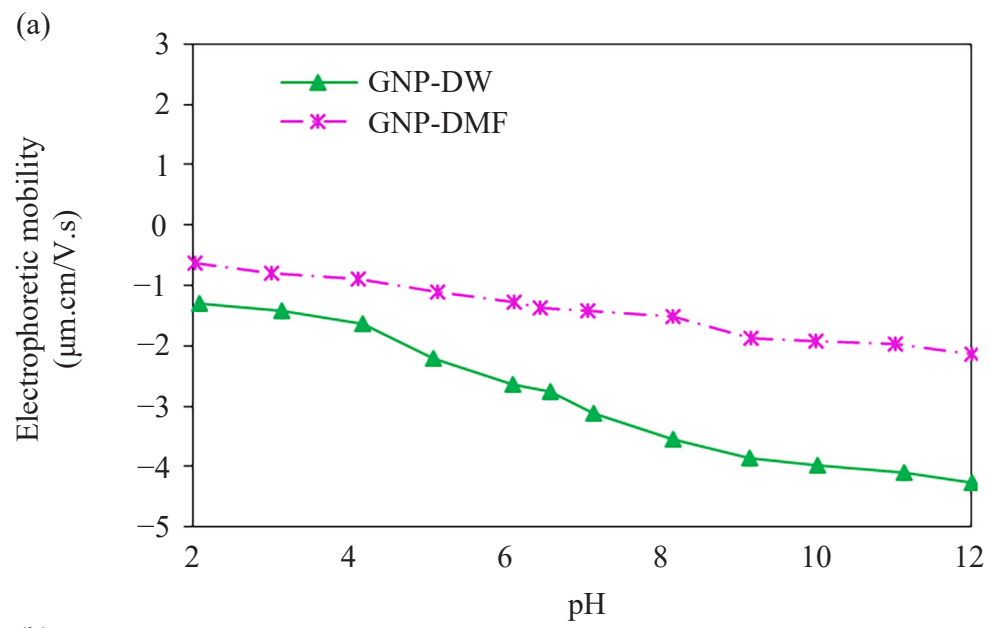

(b)

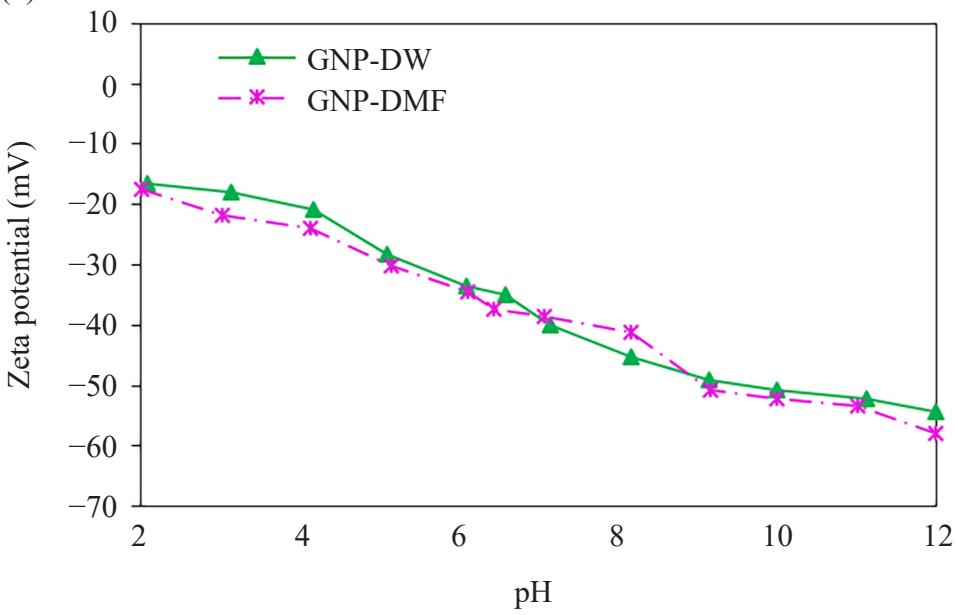

Figure 3: Illustrations of (a) electrophoretic mobility, and (b) $\xi$ potential of GNP in different suspensions (DW and DMF) observed for $\mathrm{pH} 2-12$.

\subsection{Current Profile of EPD}

Figure 4 displays the current profile versus deposition time for GNP in different suspensions (DW and DMF) with the presence of fixed applied voltage $(20 \mathrm{~V})$ and deposition time $(5 \mathrm{~min})$. The finding shows that at the fixed applied voltage of $20 \mathrm{~V}$ and deposition time of $5 \mathrm{~min}$, the current intensity of GNP-DW and GNPDMF suspensions demonstrated an increased trend in the current intensity. The GNP-DW and GNP-DMF suspensions reveal rapid movements due to their higher EM, shown in Figure 3(a), along with better dispersion and stability as supported via their highly negative $\xi$ potential, as in Figure 3(b). 
The current intensity of GNP-DW is higher compared to GNP-DMF suspension. The finding implies that the advantage of using DW with higher dielectric constant (78.54) compared to DMF (36.70), indicating that the use of lower applied voltage $(20 \mathrm{~V})$ within $5 \mathrm{~min}$ is adequate for improvement in the current intensity, leading to effective deposition of GNP. In contrast, the use of organic solvent such as DMF with lower dielectric constant compared to DW restricts the ions charge on the fillers due to inadequate dissociative power and therefore higher applied voltage is required for enhancement in the deposition of the various fillers. ${ }^{5}$

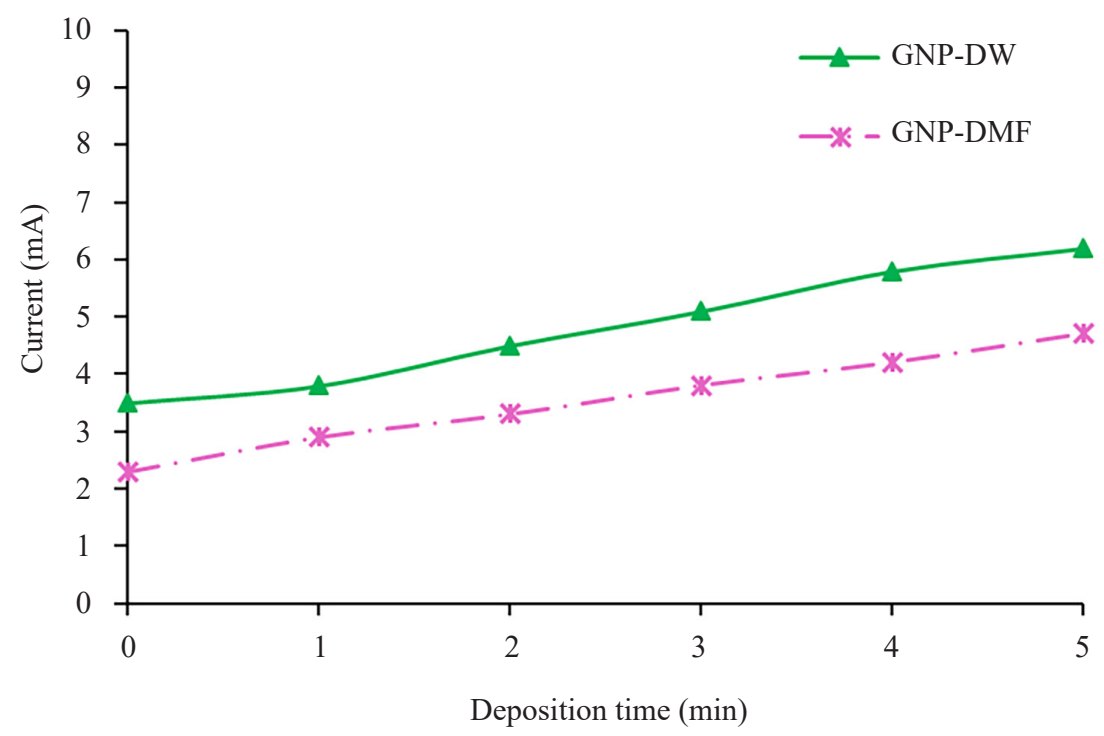

Figure 4: Current as function of deposition time via EPD of GNP-DW and GNP-DMF suspensions with the presence of fixed applied voltage $(20 \mathrm{~V})$ and deposition time $(5 \mathrm{~min})$.

\subsection{Morphologies of Deposited CF Fabric}

The FESEM images reveal the non-uniformly deposited GNP-DW onto CF with the CF surface almost completely covered with GNP, shown in Figures 5(a and b). The morphology of deposited GNP-DW reveals flake-like structure with wrinkled and aggregated at certain areas, suggesting the higher EM of GNP-DW, as in Figure 3(a), accelerating aggregated GNP onto CF. It is believed that the constant applied voltage $(20 \mathrm{~V})$ and deposition time $(5 \mathrm{~min})$ were sufficient to facilitate the movements of GNP-DW in the direction of electric fields. Therefore, the higher EM of GNP-DW due to lower viscosity of DW (0.890 mPa.s) compared to DMF (0.920 mPa.s), contributing to the higher deposition of the GNP-DW onto the CF surface. 
The deposited GNP-DMF onto CF demonstrated uniformity with slightly lower deposition of GNP, shown in Figure 5(c and d). Theoretically, the non-polar behaviour of untreated GNP being easily dispersed in polar aprotic solvent (DMF) compared to polar protic solvent (DW) is due to their low polar and hydrogenbonding strength, contributing to dispersibility and stability of the GNP-DMF suspension. It is hypothesised that the presence of fixed applied voltage of 20 $\mathrm{V}$ and deposition time of $5 \mathrm{~min}$ were insufficient to facilitate the lower EM of GNP-DMF suspension, shown in Figure 3(a). The finding revealed the lower EM of GNP-DMF suspension due to the higher viscosity of DMF (0.920 mPa.s) compared to DW (0.890 mPa.s), restricting the EM of the GNP-DMF suspension. Additionally, the lower dielectric constant of DMF (36.70) in comparison with DW (78.54) limits the ions charge on the GNP-DMF probably due to insufficient dissociative power, resulting in the slightly lower deposition of the GNP-DMF onto the CF surface. ${ }^{5}$
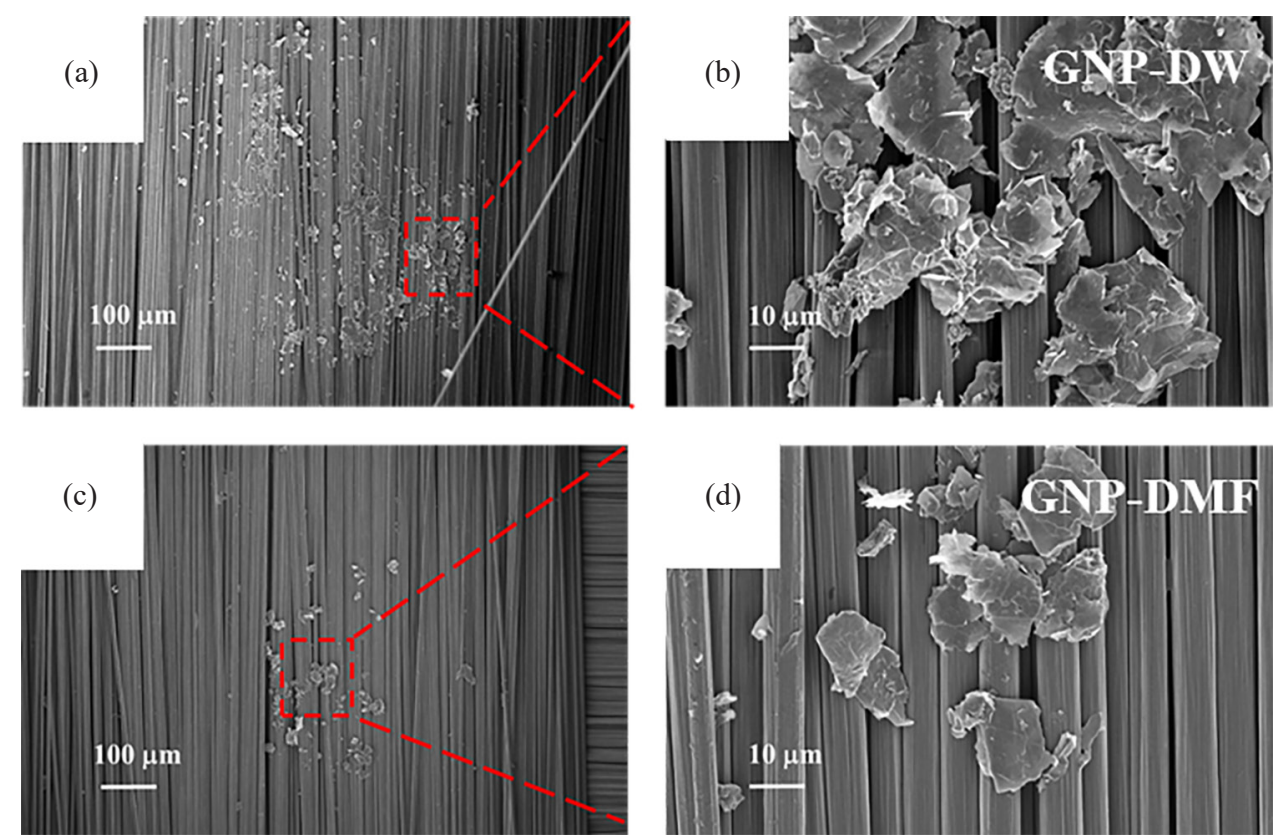

Figure 5: FESEM images of GNP in ( $a$ and b) DW, and (c and d) DMF suspensions, respectively with the presence of fixed applied voltage $(20 \mathrm{~V})$ and deposition time of 5 min (magnification of 100X for (a and c) and 1KX for (b and d). 


\subsection{Flexural Properties}

Figure 6 exhibits representative flexural stress-strain behaviour and flexural properties of CF/EP hybrid laminated composites with GNP dispersed in different suspensions (DW and DMF) deposited onto CF at fixed applied voltage $(20 \mathrm{~V})$ and deposition time $(5 \mathrm{~min})$. Based on the flexural properties behaviour, it was obvious that the deposition of GNP in different suspensions (DW and DMF) onto the CF demonstrated significant trends in the flexural properties of CF/EP hybrid laminated composites, suggesting improved interfacial interactions between fibre and matrix with reinforcing effects of the fillers. ${ }^{11}$

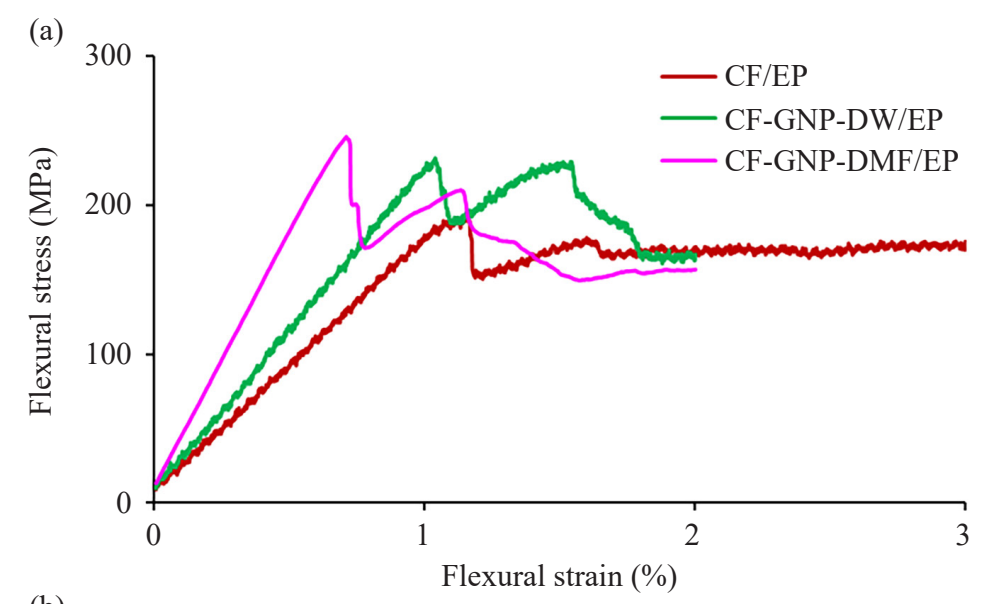

(b)

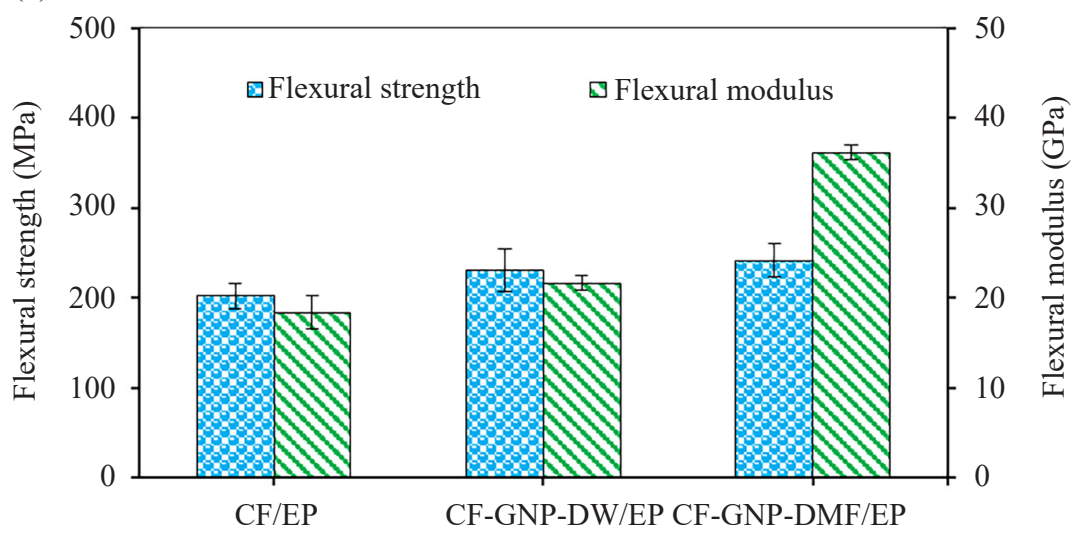

Figure 6: Illustrations of (a) representative flexural stress-strain, and (b) flexural properties of CF/EP hybrid laminated composites with GNP-DW and GNPDMF deposited onto CF at fixed applied voltage $(20 \mathrm{~V})$ and deposition time (5 min). 
Representative flexural stress-strain behaviour of CF/EP laminated composites with GNP dispersed in different suspensions (DW and DMF) deposited onto CF surface at fixed applied voltage $(20 \mathrm{~V})$ and deposition time $(5 \mathrm{~min})$ are shown in Figure 6(a). The flexural stress-strain behaviour of CF/EP hybrid laminated composites with different fillers demonstrates different slopes which were affected by different modulus. ${ }^{12}$ In particular, the flexural stress-strain curves display slightly linear deformation trend of stress to maximum value (associated with the flexural strength of CF/EP hybrid laminated composite) followed by the non-linear deformation trend with irregularities of curves, indicating the initiation of crack growth due to random fibre breakage and therefore decreases the flexural stress as fibre breakage through thickness increased. The curves show that after the crack onset, the irregularities in the flexural stress-strain curves with a yielding-like behaviour is observed as the delamination length increases likely due to bridging effect of filler. ${ }^{13}$ For the control sample, delamination occurs due to the cracking of the EP related with bridging of $\mathrm{CF}$ that acts as resistance against delamination propagation at the crack surface. Similar mechanism was observed when fillers were added onto $\mathrm{CF} / \mathrm{EP}$ hybrid laminated composites with the filler also bridging the crack surface as well as increasing resistance against crack growth. ${ }^{11}$

As revealed in Figure 6(b), the deposition of GNP-DW and GNP-DMF onto the $\mathrm{CF}$ significantly improved the flexural properties of CF/EP hybrid laminated composites. The flexural strength and modulus of the control sample (CF/ EP laminated composite) displayed $202 \mathrm{MPa}$ and $18 \mathrm{GPa}$, respectively. When compared with control sample, the findings showed that the flexural strength and modulus of different CF/EP hybrid laminated composites deposited with GNPDW suspension (CF-GNP-DW/EP) exhibited an improvement of approximately $14 \%(231 \mathrm{MPa})$ and $22 \%$ (22 GPa), respectively whereas, the other CF/EP hybrid laminated composites deposited with the GNP-DMF suspension (CF-GNP-DMF/ EP) demonstrated 19\% (241 MPa) and 100\% (36 GPa) improved in flexural strength and modulus, correspondingly. Based on these findings, it was apparent that the flexural properties of CF-GNP-DMF/EP were higher compared to CF-GNPDW/EP. An improvement in the flexural properties of CF/EP hybrid laminated composites with deposited GNP-DMF onto CF may be associated with the EPD suspension parameters (GNP size, EM, $\xi$ potential, dielectric constant, viscosity and colloidal stability) that influenced the dispersibility and stability of GNP in the liquid medium and subsequently govern the flexural properties of GNP/CF/EP hybrid laminated composites. 


\section{CONCLUSION}

This study confirms that the dispersibility and stability of GNP in the liquid medium (with and without the presence of applied voltages) were influenced by those related to the suspension such as GNP size, EM, $\xi$ potential, dielectric constant, viscosity and colloidal stability. The EM of GNP-DMF was slightly slower compared to GNP-DW due to the higher viscosity of DMF (0.92 mPa.s) compared to DW (0.89 mPa.s). The current intensity of the GNP-DMF suspension was lower than GNP-DW suspension probably due to the lower dielectric constant of DMF (36.70) compared to DW (78.54) and therefore limits the ions charge on the GNP-DMF due to insufficient dissociative power. The morphology observation of deposited GNP-DMF onto CF surface demonstrated uniformity with slightly lower deposition of GNP-DMF compared to GNP-DW, which subsequently influenced the final properties of the CF/EP hybrid laminated composites. However, the GNP$\mathrm{DMF} / \mathrm{CF} / \mathrm{EP}$ hybrid laminated composites exhibits the highest flexural properties compared to GNP-DW/CF/EP hybrid laminated composites. These findings proved that the colloidal dispersibility of GNP in DW and DMF suspensions, respectively used in EPD, govern the flexural properties of GNP/CF/EP hybrid laminated composites.

\section{ACKNOWLEDGEMENTS}

This work was supported by the Fundamental Research Grant Scheme-Malaysia Rising Stars Awards (FRGS-MRSA), grant no. 6071385 and Universiti Sains Malaysia.

\section{REFERENCES}

1. Mani, A., Tambe, P. \& Rahaman, A. (2019). Flexural properties of multiscale nanocomposites containing multiwalled carbon nanotubes coated glass fabric in epoxy/graphene matrix. Compos. Interf., 1-28, https://doi.org/10.1080/09276440 .2019.1569396.

2. Wang, C. et al. (2016). Electrophoretic deposition of graphene oxide on continuous carbon fibers for reinforcement of both tensile and interfacial strength. Compos. Sci. Technol., 135, 46-53, https://doi.org/10.1016/j.compscitech.2016.07.009.

3. Lubineau, G. \& Rahaman, A. (2012). A review of strategies for improving the degradation properties of laminated continuous-fiber/epoxy composites with carbon-based nanoreinforcements. Carbon, 50(7), 2377-2395, https://doi. org/10.1016/j.carbon.2012.01.059. 
4. Felisberto, M. et al. (2017). Carbon nanotubes grown on carbon fiber yarns by a low temperature CVD method: A significant enhancement of the interfacial adhesion between carbon fiber/epoxy matrix hierarchical composites. Compos. Commun., 3, 33-37, https://doi.org/10.1016/j.coco.2017.01.003.

5. Besra, L. \& Liu, M. (2007). A review on fundamentals and applications of electrophoretic deposition (EPD). Prog. Mater Sci., 52(1), 1-61, https://doi. org/10.1016/j.pmatsci.2006.07.001.

6. Ervina, J. et al. (2019). Colloidal stability of water-based carbon nanotube suspensions in electrophoretic deposition process: Effect of applied voltage and deposition time. Compos. A Appl. Sci., 117, 1-10, https://doi.org/10.1016/j. compositesa.2018.11.002.

7. Abdalla, M. et al. (2008). Cure behavior of epoxy/MWCNT nanocomposites: The effect of nanotube surface modification. Polym., 49(15), 3310-3317, https://doi. org/10.1016/j.polymer.2008.05.016.

8. Zhang, Q. et al. (2016). Dispersion stability of functionalized MWCNT in the epoxy-amine system and its effects on mechanical and interfacial properties of carbon fiber composites. Mater. Des., 94, 392-402, https://doi.org/10.1016/j. matdes.2016.01.062.

9. Johnson, D. et al. (2015). A manufacturing perspective on graphene dispersions. Curr. Opin. Coll. Interf. Sci., 20(5-6), 367-382, https://doi.org/10.1016/j. cocis.2015.11.004.

10. Shieh, Y.-T. et al. (2007). Effects of polarity and $\mathrm{pH}$ on the solubility of acidtreated carbon nanotubes in different media. Carbon, 45(9), 1880-1890, https://doi.org/10.1016/j.carbon.2007.04.028.

11. Borowski, E. et al. (2015). Interlaminar fracture toughness of CFRP laminates incorporating multi-walled carbon nanotubes. Polym., 7(6), 1020-1045, https://doi.org/10.3390/polym7061020.

12. Awan, F. S. et al. (2018). Interfacial mechanical properties of carbon nanotubedeposited carbon fiber epoxy matrix hierarchical composites. Compos. Interf., 25(8), 681-699, https://doi.org/10.1080/09276440.2018.1439620.

13. Ricciardi, M. R. et al. (2015). Mechanical behavior of hybrid fiber-reinforced composites manufactured by pulse infusion. Polym. Compos., 38, 2254-2260, https://doi.org/10.1002/pc.23806. 